\title{
Age may Influence Dystrophin Expression for Bone Marrow Cells Transplantation in mdx Mice
}

\author{
Chen $\mathrm{S}^{*}$, Yao X, Zen Y and Zhang W
}

Department of Neurology, First Affiliated Hospital of Zhongshan University, Guangzhou, P.R. China

\begin{abstract}
Objective: To investigate if age influence dystrophin expression for bone marrow cells (BMCs) transplantation in mdx mice.

Methods: BMCs of C57BL/6 mice(new born) $1.2 \times 10^{7}$ cells ,were injected intravenously into mdx mice of 6 weeks and 12 weeks of age, the mice were preconditioned with 8 Gy $y$ ray

Result: 12 weeks after the transplantation, $16 \%$ skeletal muscles fibers of 6 weeks and $7 \%$ those of 12 weeks age $\mathrm{mdx}$ mice have dystrophin protein expression at the sarcolemma of muscle fibers. RT-PCR and Western blot proved the results.
\end{abstract}

Conclusion: Young mdx mice transplantated with BMCs show better effect than elder one.

Keywords: mdx mice; Age; BMCs; Dystrophin protein; Expression

\section{Introduction}

Duchenne muscular dystrophy (DMD), the most common lethal genetic disorder in children, is an X-linked recessive muscle disease characterized by the absence of dystrophin at the sarcolemma of muscle fibers. The primary defect is the absence of dystrophin, a subsarcolemmal structural protein which plays an important role in maintaining the physical integrity of the muscle fibers membrane. Dystrophin, and utrophin, is part of a complex of proteins that links the internal cytoskeleton of muscle fibers to the extra cellular matrix [1]. Up to now, no method can cure the disease.

Mdx mice were setup with C57/BL10 mice, whose exon 23 of dystrophin gene was, inserted a premature stop codon [2]. By this, the dystrophin gene of $\mathrm{mdx}$ mice was knockout and dystrophin protein is absent. One of the other membrane proteins, utrophin exists. It is a wide used animal model for study DMD.

There have reported that bone marrow cells (BMCs) transplanted into dystrophic mice $(\mathrm{mdx})$ express the dystrophic protein and regenerate skeletal muscle $[3,4]$. However, the ability of BMCs expressed dytrophin protein is limited. We wonder if mice age is a variation that influences BMCs expressing dystrophic protein in $\mathrm{mdx}$ mice after transplanted. We found the expression ability of BMCs in younger mice are better and report as follow.

\section{Materials and Methods}

Mdx mice (DMD mice), purchased from The Jackson Laboratory (Maine state, America), were used in this study. C57BL/6 mice purchased from the Animal Center of Zhongshan University. All efforts were made to minimize the number of animals used and their suffering.

\section{Bone marrow cells transplantation}

Intravenous transplantation of BMCs into the body of lethally irradiated ( $8 \mathrm{~Gy} \gamma$ ray) mdx mice of two groups, 6 weeks age and 12 weeks age. BMCs are obtained from newborn C57BL/6 mice. Each mdx mice of both groups received $1.2 \times 10^{7} \mathrm{BMCs}$. Animals are then followed for 12 weeks and no changes in general health status are noted in recipient mice. The other two groups mice, six C57BL/6 and six mdx mice, served as controls. 12 weeks after the transplantation, dystrophin proteins expressing in the two groups of the mdx mice are examined.

\section{Double immunostaining of dystrophin and utrophin}

12 weeks after the transplantation, the gastrocnemius muscle of the 4 group's mice were isolated, rapidly frozen in liquid nitrogencooled isopentane, and stored at $80^{\circ} \mathrm{C}$. Unfixed sections ( $5 \mu \mathrm{m}$ thick) of frozen skeletal muscle were blocked in PBS with $1 \%$ bovine serum albumin (BSA) for 1 hour at room temperature. Then, sections were incubated with the primary antibody (dystrophin) for 1 hour at room temperature (diluted in PBS containing 1\% BSA). After three washes with PBS (5 minutes each), sections were incubated with the secondary antibody (cy3 fluorescein labeled) for 30 minutes at room temperature. Then, the sections were washed three times with PBS (10 Minutes each wash). Repeat the procedure and use utrophin antibody as primary one and secondary antibody labeled by FITC fluorescein. Finally, the sections were washed three times with PBS (10 Minutes each wash).

Slides were observed and photographed under confocal imaging system (Nicon-1). For the each transplanted mice of the two groups, observing 100 muscle fibers of one specimen continuously, and counting the dystrophin positive stained fibers. Then, counting the average positive stained fibers of each group and comparing the positive rate.

\section{Reverse transcription PCR analysis}

Total RNA of gastrocnemius muscle of each mouse of the four groups was isolated using TRIzol (Euromedex), according to

*Corresponding author: Chen, Songlin, MD, Department of Neurology, First Affiliated Hospital of Zhongshan University, Guangzhou, 510080, P.R. China, Tel: +86 15913164358; E-mail: csl071@163.com

Received October 31, 2012; Accepted December 26, 2012; Published Decembe 28, 2012

Citation: Chen S, Yao X, Zen Y, Zhang W (2012) Age may Influence Dystrophin Expression for Bone Marrow Cells Transplantation in mdx Mice. J Stem Cell Res Ther 2: 131. doi:10.4172/2157-7633.1000131

Copyright: ( 2012 Chen S, et al. This is an open-access article distributed under the terms of the Creative Commons Attribution License, which permits unrestricted use, distribution, and reproduction in any medium, provided the original author and source are credited. 
manufacturer's instructions. Semiquantitating the RNA with nucleic acid analyzer (Beckman). RT-PCR of GAPDH and dystrophin was performed with $2 \mu \mathrm{g}$ of total RNA. RNA for RT-PCR was converted to cDNA with a first-stand cDNA synthesis kit (Amersham Pharmacia Biotechnology) according to the manufacturer's recommendations. The sequences of PCR primers that amplify were: dystrophin (444 bp), forward (10662 10680), 5-CTCGTAGTCCTGCCCAGA-3, and reverse (11106 111087), 5-GTTTGACTGCCAAC2CACTCG-3. GAPDH (451bp): forward 5-ACCACAGTCCATGC2CATCAC-3, reverse 5-TCCACCACCCTGTTGCTGTA-3.

PCR was performed with TaKaRa recombinant Taq (Takara Shuzo, Kyoto, Japan) for 30 cycles, with each cycle consisting of $94^{\circ} \mathrm{C}$ for $30 \mathrm{~s}$, $62^{\circ} \mathrm{C}$ or $65^{\circ} \mathrm{C}$ for $30 \mathrm{~s}$, and $72^{\circ} \mathrm{C}$ for $20 \mathrm{~s}$, With an additional 10 -min incubation at $72^{\circ} \mathrm{C}$ after completion of the last cycle.

\section{Western blotting analysis of dystrophin and GAPDH}

12 weeks after transplantation, gastrocnemius muscle of each mice of the four groups were harvested, minced with a scissors, homogenized in a Polytron tissue, grinder for 30 seconds at a medium-range speed, and solubilized in a buffer containing $10 \mathrm{~mol} / \mathrm{L}$ Tris- $\mathrm{HCl}(\mathrm{pH} 8.0), 150$ $\mathrm{mmol} / \mathrm{L} \mathrm{NaCl}, 5 \mathrm{mmol} / \mathrm{LEDTA}, 1 \%$ Triton X-100, and $60 \mathrm{mmol} / \mathrm{L}$ octyl glucoside for 45 minutes at $4^{\circ} \mathrm{C}$. Samples were centrifuged at $13,000 \mathrm{~g}$ for 10 minutes at $4^{\circ} \mathrm{C}$ to remove insoluble debris. Soluble proteins were resolved by sodium dodecyl sulfate-polyacrylamide gel electrophoresis (SDS-PAGE) (10\% acrylamide) and transferred to nitrocellulose membranes. Blots were blocked for 1 hour in Tris-buffered saline Tween (TBST) (10 mmol/L Tris- $\mathrm{HCl}, \mathrm{pH} 8.0,150 \mathrm{mmol} / \mathrm{L} \mathrm{NaCl}, 0.2 \%$ Tween 20) Containing $4 \%$ powdered skim milk and $1 \%$ BSA. Then, the membranes were incubated for 1 hour with a given primary antibody (or an overnight incubation with the anti-dystrophin antibody), and diluted in TBST/1\% BSA. After three washes with TBST, the blots were incubated for 30 minutes with horseradish peroxidase (HRP)conjugated secondary antibodies, diluted in TBST/1\% BSA. Antibodybound proteins were detected using an enhanced chemiluminescence detection kit according to the manufacturer's instructions (Pierce, Rockford, IL). 12\% separation gel was made for GAPDH.

The comparison for the rate of dystrophin-positive stain fibers between the two groups are done by using chi square test ( $\chi 2$ test).

\section{Results}

\section{Dystrophin, utrophin double immunostaining}

When checking the dystrophin protein expressing in the transplanted mice, we do the double immunostaining of dystrophin and utrophin to the skeletal muscle of the 4 groups mice. For the C57BL/6 mice, all the membrane of the skeletal muscle fibers are positive stained with dystrophin and utrophin antibody and showed bright yellow, the mixed colour of cy3 and FITC (Figure 1A). That means dystrophin and utrophin protein show normal distribution on the membrane of the muscle fibers. For the untransplanted mdx mice, all the membrane of the skeletal muscle fibers are only positive stained with utrophin antibody and showed green, the colour of FITC (Figure 1B). That means only utrophin protein is on the membrane of skeletal muscle fibers of the untransplanted mdx mice. For the transplanted mdx mice of 8 weeks age, there are about $7 \%$ skeletal muscle fibers have positive stained with dystrophin and utrophin antibody and show bright yellow. The rest are only positive stained with utrophin antibody and showed green colour of FITC (Figure 1D). For the transplanted mdx mice of 6 weeks age, there are about 16\% skeletal muscle fibers have positive stained with dystrophin and utrophin antibody and show bright yellow. The rest are only positive stained with utrophin antibody and showed green (Figure 1C). The dystrophin positive stained skeletal muscle fibers have a tendency of clustering distribution.

The percent of dystrophin positive stained muscle fiber of $\mathrm{mdx}$ mice of 6 weeks age are more than those of 8 weeks age obviously $\left(\mathrm{X}^{2}=3.98, \mathrm{P}<0.05\right)$. That means the expression abilities of BMCs in younger mice are better.

\section{RT-PCR}

For go on prove the results, we do the RT-PCR of dystrophin gene for the four groups of mice. The results are shown in Figure 2. The expression of dystrophin gene of C57BL/6 mice is very strong, while the untransplanted $\mathrm{mdx}$ mice are negative. The expressions of the transplanted mdx of 6 weeks are stronger than those of 8 weeks mdx mice (Figure 2).

\section{Western blot}

The results of Western blot of the dystrophin protein of the muscle tissue of the four groups mice are as Figure 3. Under the same concentration, the expressions of dystrophin protein of C57 BL/6 mice are very strong, while untransplanted $\mathrm{mdx}$ mice negative. The
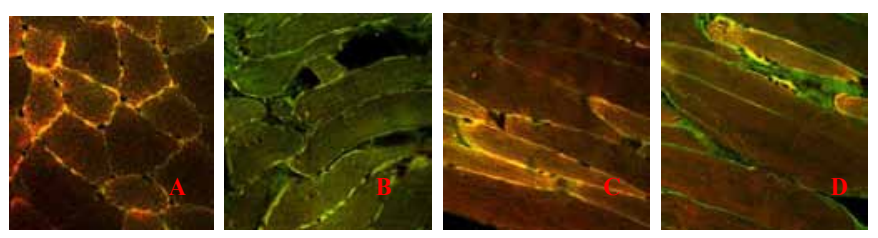

Figure 1: The confocol imaging of double stain of utrophin and dystrophin antibody of the muscle tissue of the four groups mice.

A: The confocol imaging of double stain of utrophin and dystrophin antibody of skeletal muscle piece of C57BL/6 mice; The membrane show utrophin (FITC) and dystrophin (Cy3) mixed colour: bright yellow. $(\times 400)$.

$\mathrm{B}$ : The confocol imaging of double stain of utrophin and dystrophin antibody of skeletal muscle piece of controlled mdx mice; The membrane only show the colour of utrophin: bright green. $(\times 400)$.

$\mathrm{C}$ : The confocol imaging of double stain of utrophin and dystrophin antibody of skeletal muscle piece of 6 weeks age mdx mice; $16 \%$ membrane show dystrophin and utrophin's colour : bright yellow. The rest show the colour of utrophin : bright green. $(\times 400)$.

D: The confocol imaging of double stain of utrophin and dystrophin antibody of skeletal muscle piece of 12 weeks age mdx mice; $7 \%$ membrane show dystrophin and utrophin's colour : bright yellow. The rest show the colour of utrophin: bright green. $(\times 400)$.

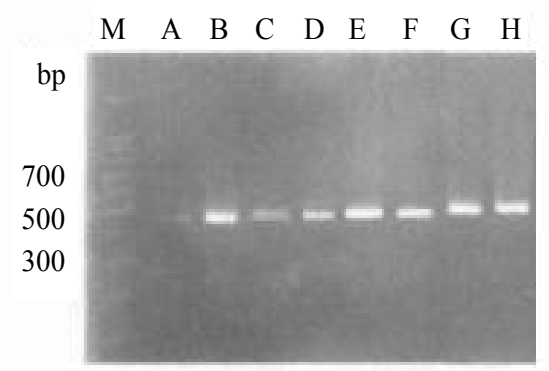

Figure 2: RT-PCR results of dystrophin and GAPDH 12 weeks after BMCs transplantation.

M: marker; A-D: expression of dystrophin; E, H: expression of GAPDH; A, E:untransplanted mdx mice. B, F: C57 BL /6 mice. C,G: mdx mice of 12 weeks age, D, H: mdx mice of 6 weeks age. 


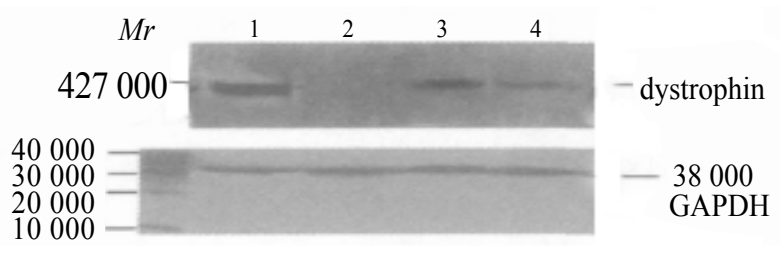

Figure 3: Western blot expression of dystrophin and GAPDH.

1. C57 BL /6 mice; 2. untransplanted mdx mice; 3.mdx mice of 6 weeks age 12 weeks after BMCs transplantation; 4.mdx mice of 12 weeks age 12 weeks after BMCs transplantation; $\mathrm{M}$ r: relative molecular mass

expressions of the transplanted $\mathrm{mdx}$ of 6 weeks are stronger than those of 8 weeks.

\section{Discussion}

BMCs have the ability of multiple differentiation and can be used to treat many disease are confirmed $[5,6]$. Studies proved that bone marrow cells transplanted into lethally irradiated $\mathrm{mdx}$ can become part of skeletal muscle myofibers and have dystrophin protein expression [3]. Human marrow cells also have this ability. However, the percent of normal skeletal muscle fibers is only about $0.5 \%-0.9 \%$ [7]. For clinical practice of BMCs in DMD patients, we should find a way, especially a physiological one, to raise the dystrophy expression.

Many methods, such as clonal isolation of muscle-derived stem cells [8]; Choosing the cell sex in stem cell for transplanted [9]; Selecting multipotent cell population from human adipose tissue [10], have been tried to raise the expression. We want to find a physiological and simple way to raise the expression of dystrophin and then serve for the clinical practice. The purpose of our experiment is to answer if age is variation for dystrophin protein expressed after BMCs transplanting in $\mathrm{mdx}$ mice. Our result show that 12 weeks after BMCs transplanted, there are $7 \%$ muscle fibers expressing dystrophin protein in the 12 weeks age $\mathrm{mdx}$ mice and $16 \%$ in the 6 weeks age ones. There are obviously different for the dystrophin protein expression between the two groups. That mean the effect of BMCs transplantation is better for younger mice. Double stain, RT-PCR, and western blot all prove the results.

Why there is such result? Two reasons may be explained for it. First, for the BMCs, the muscular tissue environment of different age $\mathrm{mdx}$ mice is different. The typical muscular tissue of $\mathrm{mdx}$ mice is normal at the neonatal stage. The first acute necrosis occurs on the three or four weeks after birth, and will persist one month. At the age of eight weeks, half of muscle appear as hyperplasia, and compensate the necrosis on the whole [11]. After eight weeks, mdx mice complete their regeneration, so the treating effect of BMCs is decrease. CK concentration of serum can reflect the injury level of the membrane of skeletal muscle fibers, and reflect the pathologic change of the muscle tissue indirectly. The CK level of DMD patients increase continually before ten years old age and decrease after then. These reflect the course of hyperplasia and necrosis of skeletal muscle fibers [12]. So maybe the age of ten for DMD patients, just like the 8 weeks mdx mice, is the critical for BMCs transplantation. So, for DMD patients, does the transplantation before ten years old maybe show better effect? The skeletal muscle tissues of advanced DMD patients are degeneration, necrosis and fibrosis. The transplanting effect is not so good of course. Secondly, for the different age mice, the hormone concentration in their body is different. Researchers find the concentration of hormone is different in the serum of different age groups [13]. The concentrations of the hormones related to growth are different in the body of different age mice, so the growth and reproduced ability of their muscle fibers are also different. By the effects of those hormones, BMCs may grow better in young mice body. So the transplanting effect is better. There is also report that, when BMCs transplantation is used for the treating of Niemann-Pick disease, their effect for young mice is better than adult [14].

For BMCs transplanting in DMD patients, the basic problem is finding a better, easy method to raise the absent protein expressing and improve function of muscle tissue. Answering the questions, such as how many times and how much number of BMCs transplanted are most favorable for the absent protein expression, and will be important in clinic applications of BMCs.

12 weeks after transplantation with BMCs, more dystrophin protein expressed in the body of young mdx mice and there maybe similar result in human being.

\section{Acknowledgement}

This research was supported by The Medical Researching Fund of Guangdong Province, P.R. China (No. A2006177) and Natural Science Fund of Guangdong Province, P.R. China (No. 06300815).

\section{References}

1. Worton R (1995) Muscular dystrophies: diseases of the dystrophin-glycoprotein complex. Science 270: 755-756.

2. Bulfield G, Siller WG, Wight PA, Moore KJ (1984) X-chromosome-linked muscular dystrophy (mdx) in the mouse. Proc Natl Acad Sci USA 81: 11891192.

3. Gussoni E, Soneoka Y, Strickland CD, Buzney EA, Khan MK, et al. (1999) Dystrophin expression in the $\mathrm{mdx}$ mouse restored by stem cell transplantation. Nature 401: 390-394.

4. Sohn RL, Gussoni E (2004) Stem cell therapy for muscular dystrophy. Expert Opin Biol Ther 4:1-9.

5. Minguell JJ, Erices A, Conget P (2001) Mesenchymal stem cells. Exp Biol Med 226: $507-520$.

6. Herzog EL, Chai L, Krause DS (2003) Plasticity of marrow-derived stem cells. Blood 102: 3483-3493.

7. Gussoni E, Bennett RR, Muskiewicz KR, Meyerrose T, Nolta JA, et al. (2002) Long-term persistence of donor nuclei in a Duchenne muscular dystrophy patient receiving bone marrow transplantation. J Clin Invest 110: 807-814.

8. Lee JY, Qu-Petersen Z, Cao B, Kimura S, Jankowski R, et al. (2000) Clonal Isolation of Muscle-derived Cells Capable of Enhancing Muscle Regeneration and Bone Healing. J Cell Biol 150: 1085-1100.

9. Deasy BM, Lu A, Tebbets JC, Feduska JM, Schugar RC, et al. (2007) A role for cell sex in stem cell-mediated skeletal muscle regeneration: female cells have higher muscle regeneration efficiency. J Cell Biol 177: 73-86.

10. Rodriguez AM, Pisani D, Dechesne CA, Turc-Carel C, Kurzenne JY, et al (2005) Transplantation of a multipotent cell population from human adipose tissue induces dystrophin expression in the immunocompetent mdx mouse. $J$ Exp Med 201: 1397-1405.

11. Stedman HH, Sweeney HL, Shrager JB, Maguire HC, Panettieri RA, et al (1991) The mdx mouse diaphragm reproduces the degenerative changes of Duchenne muscular dystrophy. Nature 352: 536-539.

12. Konagaya M, Takayanagi T (1986) Regularity in the change of serum creatine kinase level in Duchenne muscular dystrophy. A study with long-term follow-up cases. Jpn J Med 25: 2-8.

13. Guo SX, Li YS, Tian D (1997) The effect of estrogen on regulation of tubulin synthesis and life span in mice. Chinese J Geriatrics 6: 233-235.

14. Miranda SR, Erlich S, Visser JW, Gatt S, Dagan A, et al. (1997) Bone marrow transplantation in acid sphingomyelinase-deficient mice: Engraftment and cell migration into the brain as a function of radiation, age, and phenotype. Blood 90: 444-452. 